\title{
Synthesis of Spherical Carbon Nanoparticles and their EDLC Properties
}

\author{
Guo-Bin Zheng ${ }^{1, a}$, Hideaki Sano ${ }^{2, b}$ and Yasuo Uchiyama ${ }^{3, c}$ \\ ${ }^{1,2,3}$ Department of Materials Science and Engineering, Nagasaki University, \\ Bunkyo machi 1-14, Nagasaki, Japan \\ agbzheng@nagasaki-u.ac.jp, bsano@nagasaki-u.ac.jp, 'uchiyama@nagasaki-u.ac.jp
}

Keywords: Carbon nanoparticles, EDLC, Sol-gel.

\begin{abstract}
Spherical carbon nanoparticles were synthesized by sol-gel polymerization of resorcinol and formaldehyde in the presence of $\mathrm{CTAB}$ (cetyltrimethylammonium bromide) and subsequent carbonization at $900^{\circ} \mathrm{C}$. It is found that the amount of CTAB, water and catalyst showed a significant impact on the morphology and size of carbon nanoparticles. According to the BET surface area and capacitance, the nanoparticles were divided into two groups. The carbon nanoparticles with surface area of $725-758 \mathrm{~m}^{2} / \mathrm{g}$ showed capacitance between $120-140 \mathrm{~F} / \mathrm{g}$, while those with surface area of $596-649 \mathrm{~m}^{2} / \mathrm{g}$ showed capacitance only $60-80 \mathrm{~F} / \mathrm{g}$. Difference in the pore size of $1.5 \mathrm{~nm}$ to $3.0 \mathrm{~nm}$ probably contributed to the difference of capacitance.
\end{abstract}

\section{Introduction}

Electrical double layer capacitors (EDLCs) are expected to play an auxiliary role to batteries in electrical vehicles, energy storage devices for wind power or solar power, since EDLCs possess high power and rapid charge/discharge characteristics but much lower energy density than batteries [1]. The aim of development of EDLCs is to raise their energy density without sacrificing their high power and rapid charge/discharge characteristics. The performance of EDLCs depends largely upon the porous carbon electrode, which allows formation of high surface electrical double layer in the electrode/electrolyte interface [2]. Though high surface area is beneficial to the energy density, the pores, which provide path for transportation of ions, play important role especially when charge/discharge at high current. Thus activated carbon with high surface area and only micropores failed to show high performance at high charge/discharge rate because of lack of mesopores. Many recent researches are focused on the development of carbon electrodes with high amount of mesopores[3,4]. This, in turn, may decrease the surface area.

A different idea is proposed in this paper, that porous carbon electrode consisted of spherical carbon nanoparticles (SCN). In such electrode of carbon nanoparticles, the space between the SCN acts as a tank of electrolytes, which provide ions for the micropores inside the SCN when charged. Since the SCNs are uniform in size, the transportation distance of ions in the SCNs should be less than the radius of SCNs. Therefore, such electrodes of SCNs should show a high performance in EDLCs.

Sol-gel polymerization of resorcinol and formaldehyde (RF) and subsequent carbonization had been used to synthesize carbon aerogel [5-7]. The addition of surfactant such as CTAB in RF solution could prevent RF gel shrinkage on drying. It is also found that spherical carbon nanoparticles could be synthesized using this process.

Here we reported the synthesis of SCNs using a sol-gel method at the presence of surfactant and the capacitance of the SCN electrodes.

\section{Experimental}

Surfactant CTAB, resorcinol, formaldehyde, $\mathrm{Na}_{2} \mathrm{CO}_{3}$ were dissolved in deionized water according to the molar ratio given in Table 1 . The mixture solutions were sealed and heated at $85^{\circ} \mathrm{C}$ and kept for 1 hour while stirred rigorously. The obtained RF gels were put in drying oven at $85^{\circ} \mathrm{C}$ for $24 \mathrm{~h}$. The dried RF gels were carbonized in $\mathrm{N}_{2}$ at $900^{\circ} \mathrm{C}$ for $1 \mathrm{~h}$, and the obtained carbon nanoparticles were denoted respectively as RFC1, RFC2, and so on as shown in Table 1. 
The carbon nanoparticles were observed using FE-SEM. Their surface area and pore distribution were measured using nitrogen adsorption. Electrodes for cyclic voltammetry (CV) and charge/discharge tests were prepared by pressing the paste of carbon nanoparticles and 5\% PTFE onto platinum mesh. $\mathrm{CV}$ tests were performed in a 3-electrode cell with $\mathrm{Ag} / \mathrm{AgCl}$ electrode as reference, and platinum as counter electrode, $1 \mathrm{M}$ $\mathrm{H}_{2} \mathrm{SO}_{4}$ solution as electrolyte in sweep rate of $5,10,20,50 \mathrm{mV} / \mathrm{s}$. The cells for

Table 1 The molar ratio of raw materials and surface area of the samples.

\begin{tabular}{lccccc}
\hline Sample & $\mathrm{H}_{2} \mathrm{O}$ & CTAB & $\mathrm{HCHO}$ & Resorcinol & $\mathrm{Na}_{2} \mathrm{CO}_{3}$ \\
\hline RFC1 & 83 & 0.041 & 2 & 1 & 0.016 \\
$\mathrm{RFC2}$ & 83 & 0.055 & 2 & 1 & 0.016 \\
$\mathrm{RFC} 3$ & 83 & 0.069 & 2 & 1 & 0.016 \\
$\mathrm{RFC} 4$ & 83 & 0.082 & 2 & 1 & 0.016 \\
$\mathrm{RFC5}$ & 83 & 0.082 & 2 & 1 & 0.032 \\
$\mathrm{RFC6}$ & 55 & 0.041 & 2 & 1 & 0.016 \\
RFC7 & 111 & 0.041 & 2 & 1 & 0.016 \\
\hline
\end{tabular}
charge/discharge tests were composed of two carbon/Pt electrodes. The charge/discharge tests of the 2-electrode cell were performed in current density of $0.1,0.2,0.4,1.0,2.5 \mathrm{~A} / \mathrm{g}$, respectively. The capacitance was then calculated according to the following equation,

$$
C=\frac{4 \times I \times \Delta t}{m \times \Delta V}
$$

where $I$ is charge current, $m$ is the mass of carbon in the two electrodes, and $\Delta V / \Delta t$ is the slope of charge curve between 0.2 and $0.6 \mathrm{~V}$, in which $V$ is the potential and $t$ is charging time.

\section{Results and discussion}

Morphology of carbon nanoparticles. Without addition of surfactant CTAB, the sol-gel polymerization of resorcinol and formaldehyde resulted in a transparent and red RF gel, but the gel shrank severely on drying at ambient pressure, turning to a hard block without pores. By addition of CTAB, white pink and opaque RF gels were obtained. They shrank slightly on drying at ambient pressure and could be easily crumbled after drying. After carbonization, carbon particles with high volume were obtained. The carbon yield of carbonization was about $40-45 \%$.
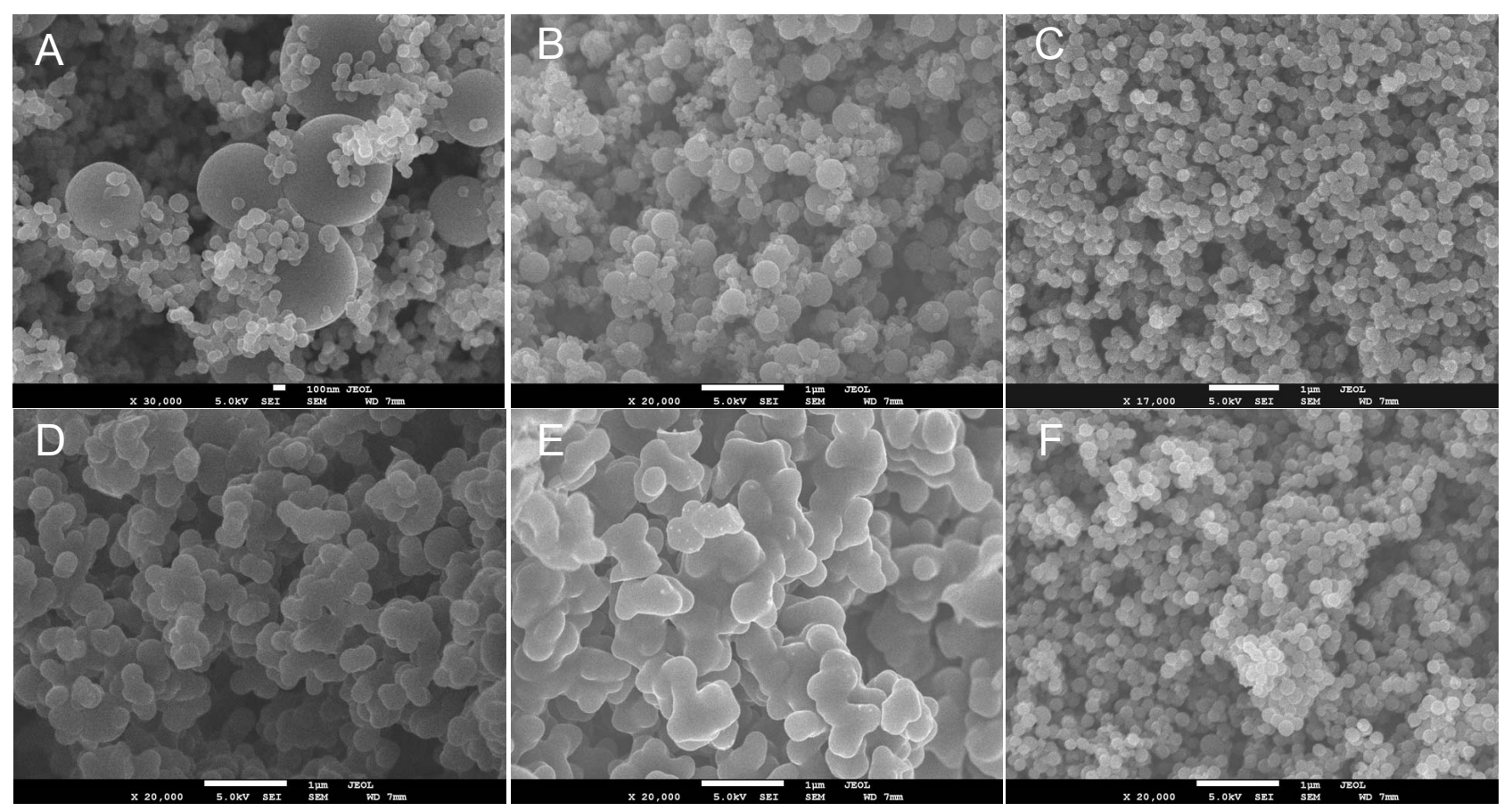

Figure 1 SEM images of RFC1(A), RFC2(B), RFC4(C), RFC5(D), RFC6(E), RFC7(F). 
Figure 1 shows the SEM images of these carbon particles. RFC1 consisted of two kinds of spherical particles, large particles with diameter of $0.8 \mu \mathrm{m}$ and small particles with diameter of 100 $\mathrm{nm}$. Since carbon particles inherited their shape from RF particles, the original RF particles have two different kinds of particles. The small particles have a narrow size distribution, indicating the size and shape of small RF particles were well controlled. Apparently, growth of some RF particles was not under control, resulting in large particles. With increasing CTAB in solution, RFC 2 and RFC3 also showed two kinds of particles with diameter of $60 \mathrm{~nm}$ and $0.4 \mu \mathrm{m}$. With further increase of CTAB in solution, the RFC4 composed of uniform particles with diameter of $200 \mathrm{~nm}$. It is believed that during the sol-gel reaction of resorcinol and formaldehyde, the surfactant was adsorbed on the surface of RF nuclei and was thus able to control the shape of RF particles. For RFC1, 2, 3, the amount of surfactant is not enough for complete coverage around the RF particles, thus many RF particles grew fast, resulting in the inhomogeneous particles. With enough CTAB as the case of RFC4, all the RF particles were covered by surfactant molecules; consequently, uniform particles were obtained.

By keeping CTAB constant and lowering the water amount, that is, concentrations of all the agents were increased, the carbon particles in RFC6 showed morphology of aggregated particles in which several primary particles aggregates to become irregular secondary particles. On the contrary, with increasing water in solution, that is, as the concentration of agents was lowered, the particles were uniform in diameter of about 100 $\mathrm{nm}$ as shown in RFC7. The higher concentration made the sol-gel reaction faster, and the distance of RF particles shorter; consequently, the RF particles were easier to collide to form aggregates. The lower concentration made the sol-gel reaction slower, thus growth of RF particles could be easily controlled.

If $\mathrm{Na}_{2} \mathrm{CO}_{3}$, the catalyst for reaction of resorcinol and formaldehyde, was doubled in solution, RFC5 showed morphology of aggregated particles, similar with that of RFC6. $\mathrm{Na}_{2} \mathrm{CO}_{3}$ played a role as catalyst in the sol-gel polymerization of RF. More $\mathrm{Na}_{2} \mathrm{CO}_{3}$ in solution increased the polymerization rate of RF, resulting in aggregation of RF particles.

Surface area and pore distribution. The BET surface area of RFCs was shown in Table 2. The surface area was between $596-758 \mathrm{~m}^{2} / \mathrm{g}$, slightly larger than those reported by Refs [5-7]. According to the value of BET surface area, the RFCs could be divided into two groups: RFC2, RFC4 and RFC6 that have surface area between 596-659 $\mathrm{m}^{2} / \mathrm{g}$, and RFC1, RFC3, RFC5 and RFC7 that have surface area between $725-758 \mathrm{~m}^{2} / \mathrm{g}$. The
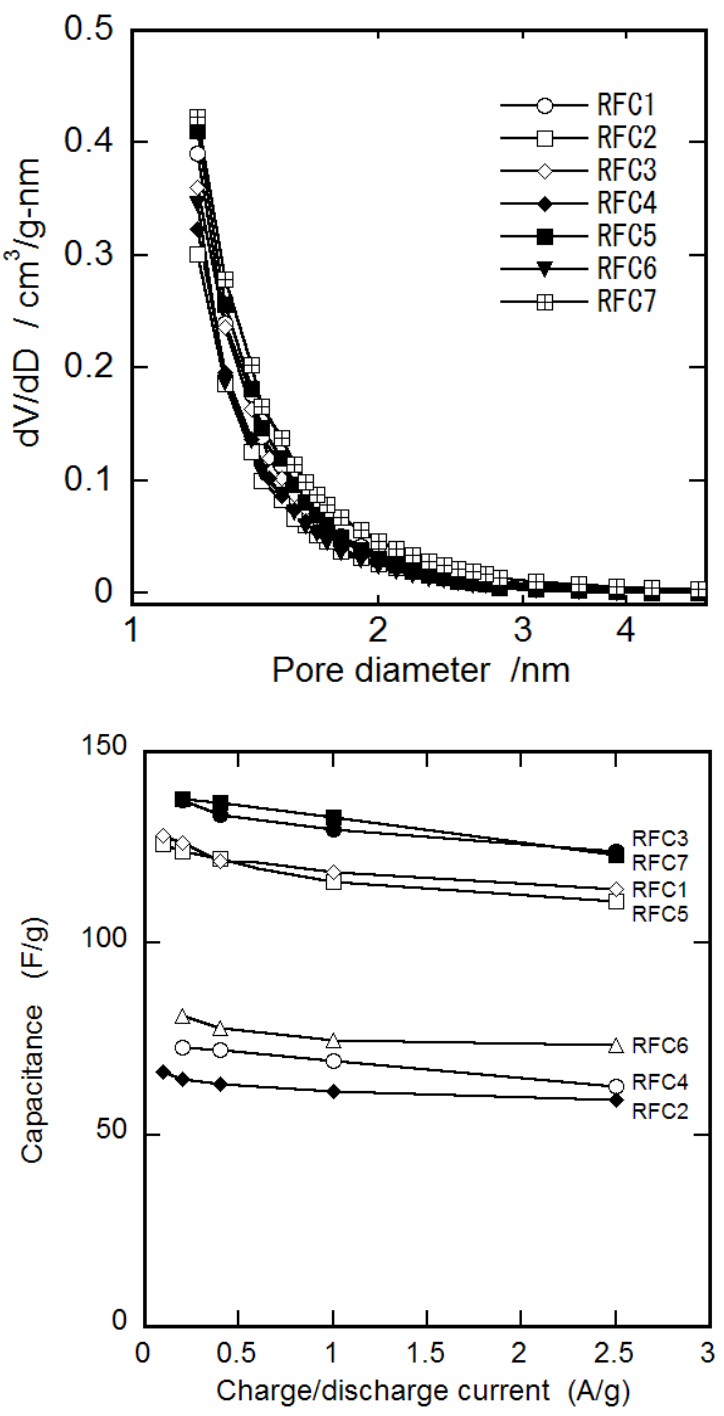

Figure 3 Relation of specific capacitance with charge/discharge current. pore distribution was shown in Figure 2. It is found that almost no mesopore larger than $3 \mathrm{~nm}$ was in the carbon particles, and only small amount of pores with size of 2 to $3 \mathrm{~nm}$. The RFC1, 3, 5, 7 showed larger volume of pores between $1.2 \mathrm{~nm}$ to $3 \mathrm{~nm}$. Except the interparticle macropores as shown in Figure 1, most of the pores are micropores smaller than $2 \mathrm{~nm}$ in the carbon nanoparticles. 
Capacitance. Figure 3 shows the dependence of capacitance of the RFCs with charge/discharge current. With increasing charge/discharge current, all the capacitance decreased only slightly. From the capacitance value, the RFCs can be divided into two groups. RFC1, 3, 5, and 7 had capacitance between 120-140 F/g, while RFC2, 4, 6 had capacitance between $60-80 \mathrm{~F} / \mathrm{g}$, almost half of the former. From the shape of the RFCs, no hint was obtained about the difference of the two groups. Therefore, the capacitance must be mainly affected by the inner structure of carbon particles, that is, pores and surface area. The capacitance of RFCs is consistent to the value of BET surface area by which the RFCs could be divided into same two groups. If considering only BET surface area, the capacitance of the group1 should be 10-20\% higher than group2. From Table 2, the specific capacitance per unit surface area of group1 is significantly higher than that of group2. From the pore distribution, group1 have larger volume of pores in 1.2-3 nm, which may benefit the capacitance. However, the reason is not clearly known yet.

Table 2 The surface area and specific capacitance of RFCs.

\begin{tabular}{llllllll}
\hline & RFC1 & RFC3 & RFC5 & RFC7 & RFC2 & RFC4 & RFC6 \\
\hline $\mathrm{S}_{\text {BET }}\left(\mathrm{m}^{2} / \mathrm{g}\right)$ & 758 & 725 & 749 & 749 & 596 & 659 & 619 \\
Capacitance $(\mathrm{F} / \mathrm{g})$ & 125 & 137 & 124 & 137 & 64 & 72 & 81 \\
Capacitance $\left(\mathrm{F} / \mathrm{m}^{2}\right)$ & 0.164 & 0.188 & 0.165 & 0.183 & 0.107 & 0.109 & 0.131 \\
\hline
\end{tabular}

For all the samples, CV curves were near rectangular at the sweeping rate of $20 \mathrm{mV} / \mathrm{s}$ or below. If the sweep rate was $50 \mathrm{mV} / \mathrm{s}$, the $\mathrm{CV}$ curves slanted a little. As a whole, the RFCs showed fast charge/ discharge properties.

Although only a few mesopores existed in carbon particles, they showed fast charge/discharge properties. From SEM observation, there are large spaces between carbon particles. The electrolyte solution in the interparticle space acts as ion tank for the micropores in the particles. On charge/discharge, the ions diffuse into or out of the particles. The diffusion path is smaller than the radius of the particles. From the result that the capacitance and charge/discharge properties did not depend on size and shape of carbon nanoparticles, it suggested that the micropores are mainly near the surface of particles.

\section{Summary}

Carbon nanoparticles were synthesized using sol-gel polymerization of resorcinol and formaldehyde with addition of surfactant CTAB. When the amount of CTAB is low, nanoparticles consisted of two kinds of particles: large particles and small particles. With the increase of CTAB amount or higher water amount, the particle size tends to become uniform. Less water and higher catalyst amount resulted in aggregated particles.

All the carbon nanoparticles showed fast charge/discharge characteristics, but no apparent relation between the capacitance and morphology or size of SNC was found. The carbon nanoparticles with surface area of $725-758 \mathrm{~m}^{2} / \mathrm{g}$ showed capacitance between $120-140 \mathrm{~F} / \mathrm{g}$, while those with surface area of $596-649 \mathrm{~m}^{2} / \mathrm{g}$ showed capacitance only $60-80 \mathrm{~F} / \mathrm{g}$. Difference in the pore size of $1.5 \mathrm{~nm}$ to $3.0 \mathrm{~nm}$ probably contributed to the difference of capacitance.

\section{References}

[1] A. Burke, Journal of Power Sources, 91 (2000) p37.

[2] R. Kötz, M. Carlen, Electrochim. Acta, 45 (2000) p 2483.

[3] W. Xing, S. Z. Qiao, R. G. Ding, et al, Carbon, 44 (2006) p 216.

[4] A. B. Fuertes, G. Lota, T. A. Centeno, E. Frackowiak, Electrochim. Acta, 50 (2005) p 2799.

[5] D. Wu, R. Fu, M. S. Dresselhaus, G. Dresselhaus, Carbon, 44 (2006) p 675.

[6] K. T. Lee, S. M. Oh, Chem. Commun. (2002) p 2722.

[7] G. Qin, S. Guo, Carbon, 39 (2001) p1935. 